\title{
Designing Feeding Networks with CORPS: Coherently Radiating Periodic Structures
}

\author{
Diego Betancourt, Carlos del Río Bocio \\ Public University of Navarra \\ Arrosadia Campus s/n, 31006 Pamplona, Spain \\ Email: carlos@unavarra.es
}

\begin{abstract}
The design of feeding networks in phased array systems results tricky since all the complex excitations should be provided to all the radiating elements of the system. Where the number of radiating elements increases the complexity of the feeding network also increase significantly. In this paper we study the possibility to apply the concepts of CORPS structures to the feeding network, obtaining a general feeding network design where the excitations should be introduced before the network and not after, reducing strongly the number of phase shifters and attenuators needed to electronically scan the beam.
\end{abstract}

Keywords: Phase array, Beam-forming Network.

\section{INTRODUCTION}

The CORPS (Coherently Radiating Periodic Structures) concept has been presented in several conferences [1] [2] applied directly to the radiating elements, but it can also be applied to the feeding network itself.

The idea is to define a horizontal (transversal to the main radiating direction) stop-band filter thanks to the periodicity of the structure in that plane. By properly interconnecting several layers of this periodic structure, the information of the input ports could be effectively driven to the output ports, exciting the radiating elements with Gaussian amplitude taper and a linear phase distribution along the structure.

The resulting feeding network is a periodic structure, easy to design it since the design procedure is reduced to a period (unit cell) of the total structure, and easy to fabricate since it can be performed modularly.

\section{FEED NETWORK DESIGN}

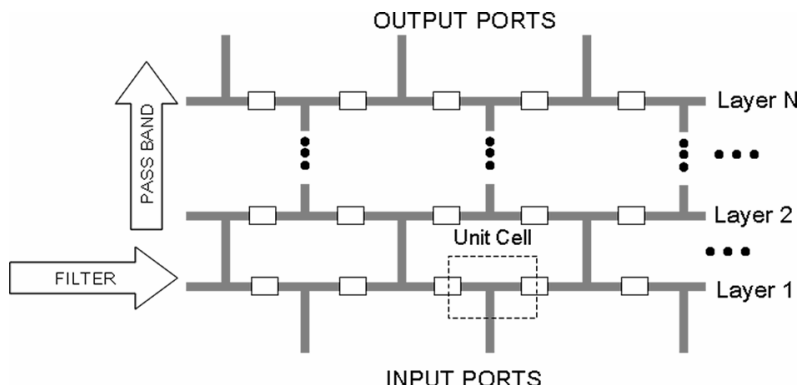

Fig. 1. Schematic of Phased Array Feed Network proposed.

In order to design Feeding networks with CORPS we must consider its schematic representation, shown in fig. 1, use the conservation of energy and take into count that the network consists only on lossless passive components. As a result, the behavior of the designed beam-forming network can be strictly related to the characteristics of the named Split and Recombination Nodes (see Fig. 2).

Split Nodes are such that have one input port and $\mathrm{N}$ output ports. At each output port is delivered a Nth part of the power introduced at input port, as can be corroborated using the following expression [3]:

$$
W_{s}=\sum_{k=1}^{N}\left[E_{k} e^{\left(j \theta_{k}\right)}\right]^{2} G_{s}
$$


Here, $\boldsymbol{W}_{\boldsymbol{S}}$ is the power delivered at the $N$ output ports of Split node, and $\boldsymbol{G} \boldsymbol{s}$ is the real part of the admittance seen at output ports. In the same way, the Recombination nodes are such that have one output port ( $N$ equal to 1$)$ and more than one input port. The power at output port can be calculated, using:

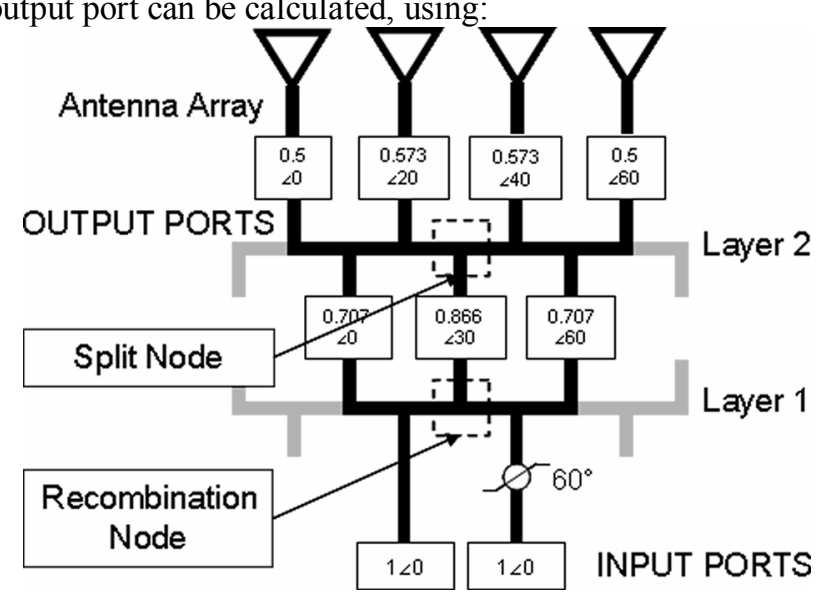

Fig. 2. Power flux throughout the structure. 2 in-ports with a phase shift of $60 \mathrm{deg}$. The phases at the output ports are a lineal representation of phase at input ports.

$$
W_{R}=\sum_{k=1}^{N}\left[E_{k, 1} e^{\left(j \theta_{k, 1}\right)}+E_{k, 2} e^{\left(j \theta_{k, 2}\right)}\right]^{2} G_{k}
$$

After doing some simplifications on (2), the power at output ports of a Recombination Node, can be expressed as.

$$
\begin{aligned}
& W_{R}=G_{R}\left[\sum_{k=1}^{N}\left(E_{k, 1}\right)^{2}+\sum_{k=1}^{N}\left(E_{k, 2}\right)^{2}\right. \\
& \left.+2 \sum_{k=1}^{N} E_{k, 1} E_{k, 2} \operatorname{Cos}\left(\theta_{k, 1}-\theta_{k, 2}\right)\right]
\end{aligned}
$$

With, $G_{R}$ one half of $G_{s}$. In Fig 2 is considered a beam-forming network of two layers, in which we set up initially two input ports of unitary amplitude, but with different phase, this is:

Input Port 1: $A e^{(j \theta)}$, and Input Port 2: $A e^{(j(\theta+\Delta \theta))}$.

Where $\mathrm{A}$ is 1 and $\Delta \theta$ is the phase shifting between ports (in this case $60 \mathrm{deg}$ ). The power delivered at output port on layer 1 and 2 is calculated using (1) and (3). From this figure we can see the lineal behavior of the phase and its direct relation with $\Delta \theta$. In general, the phase difference, between input port 1 and input port 2 , is translated to a lineal combination at output ports with initial phase equal to port 1 and final phase equal to port 2 .

\section{FEED NETWORK SIMULATION}

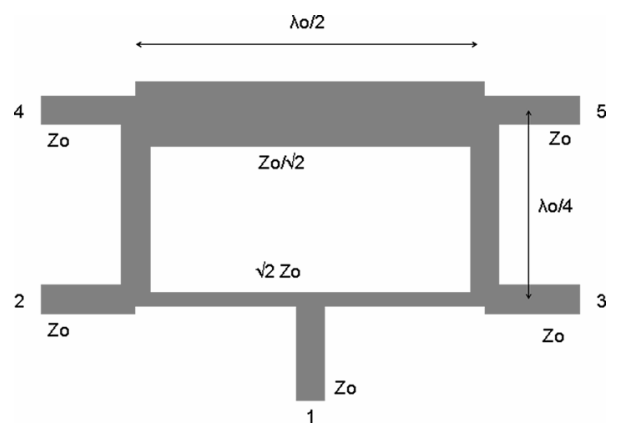

Fig. 3. Gysel Power Divider. 
One of the more important challenges at the time of implement a feed network based on CORPS principles (as shown in Fig. 2), is to found a unit cell with the desired characteristics, to know: Strong isolation between input ports and equal in-phase power delivery between layers.

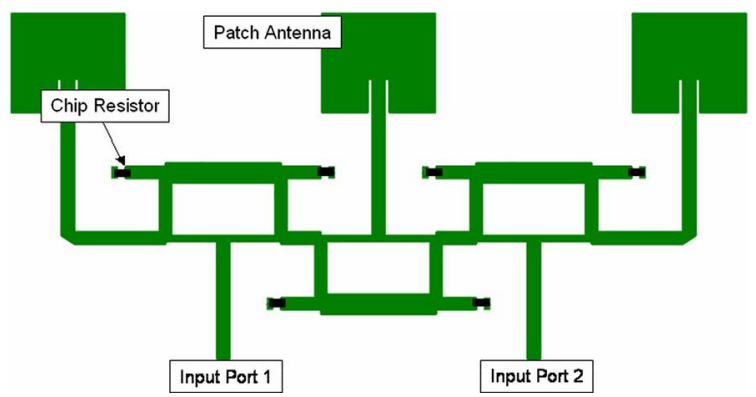

Fig. 4. Implementation of a 1 layer Beam Forming Network using Gysel power Dividers.

In order to achieve these requirements for the case of a 2D structure, based on micro-strip technology, we use a Gysel Power divider as is shown in Fig. 3. A Gysel cell [4] is a 3 $\lambda / 2$ ring impedance transformer that delivers equal in-phase power ratio in ports 2 and 3, and isolate ports 4 and 5. Thus, with a Gysel Power divider, used as unit cell of our structure, is feasible to obtain an input port isolation of more than $-30 \mathrm{~dB}$. This behavior is not obtained by the filtering effect of the periodic structure as it was initially desired of a CORPS structure, and this will be translated in a power loss when the Gysel cell in unbalanced. Nevertheless the proposed configuration could be useful to verify the behavior of a feeding network using dividers and combiners as a CORPS structure does.

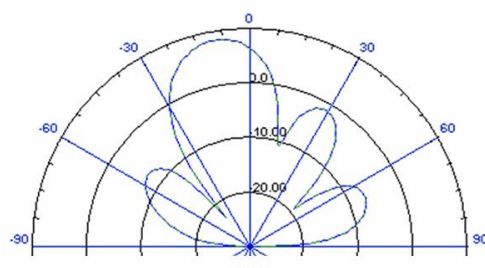

(a)

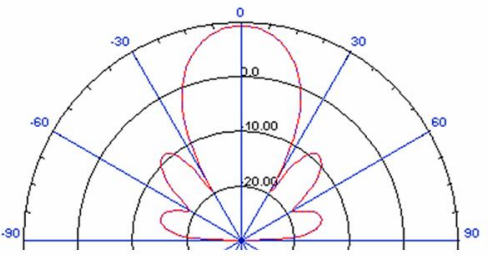

(b)

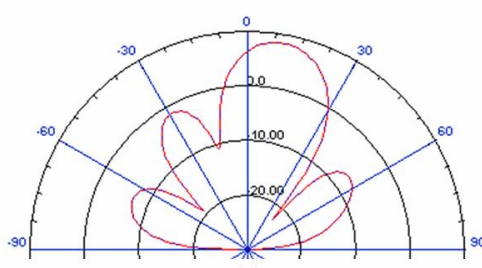

(c)

Fig. 5. Radiation Pattern for a 1 layer beam-forming Network (Implemented) with $\mathrm{d}=0.8 \lambda$ o. a) $\Delta \theta=-90$ deg, b) $\Delta \theta=0$ deg, c) $\Delta \theta=+90$ deg. Scanning angle of about $20 \mathrm{deg}$.

A beam-forming network of one layer, with the characteristics exposed before is shown in fig. 4. This network is made using Gysel Power dividers and, as radiators, a set of 3 rectangular micro-strip patch antennas.

\begin{tabular}{|c|c|c|c|c|c|c|c|c|c|c|}
\hline \multirow{2}{*}{$\begin{array}{c}\Delta \mathrm{B} \\
(\mathrm{deg})\end{array}$} & \multicolumn{3}{|c|}{$\begin{array}{l}\text { Ideal BFN out-ports [Mag / } \\
\text { Phase(deg)] }\end{array}$} & \multicolumn{3}{|c|}{$\begin{array}{c}\text { Implemented BFN out-ports [Mag } \\
\text { /Phase(deg)] }\end{array}$} & \multirow{2}{*}{$\begin{array}{l}\text { Phase out- } \\
\text { port } 1 \text { to } 3 \\
\text { (deg) }\end{array}$} & \multirow{2}{*}{$\begin{array}{l}\text { Ideal power } \\
\text { delivered }\end{array}$} & \multirow{2}{*}{$\begin{array}{l}\text { Implemented } \\
\text { power } \\
\text { delivered }\end{array}$} & \multirow{2}{*}{$\begin{array}{c}\text { Power } \\
\text { difference } \\
(\%)\end{array}$} \\
\hline & 1 & 2 & 3 & 1 & 2 & 3 & & & & \\
\hline \multirow{2}{*}{0} & 0,71 & 1,00 & 0,71 & 0,68 & 0,94 & 0,68 & \multirow{2}{*}{0,03} & \multirow{2}{*}{2,00} & \multirow{2}{*}{1,81} & \multirow{2}{*}{9,40} \\
\hline & 0,00 & 0,00 & 0,00 & 55,02 & 54,19 & 54,99 & & & & \\
\hline \multirow{2}{*}{30} & 0,71 & 0,97 & 0,71 & 0,68 & 0,91 & 0,68 & \multirow{2}{*}{30,08} & \multirow{2}{*}{1,93} & \multirow{2}{*}{1,75} & \multirow{2}{*}{9,33} \\
\hline & 0,00 & 15,00 & 30,00 & 55,10 & 39,20 & 25,02 & & & & \\
\hline \multirow{2}{*}{45} & 0,71 & 0,92 & 0,71 & 0,68 & 0,87 & 0,68 & \multirow{2}{*}{45,09} & \multirow{2}{*}{1,85} & \multirow{2}{*}{1,68} & \multirow{2}{*}{9,26} \\
\hline & 0,00 & 22,50 & 45,00 & 55,18 & 31,70 & 10,08 & & & & \\
\hline \multirow{2}{*}{60} & 0,71 & 0,87 & 0,71 & 0,67 & 0,82 & 0,68 & \multirow{2}{*}{60,09} & \multirow{2}{*}{1,75} & \multirow{2}{*}{1,59} & \multirow{2}{*}{9,14} \\
\hline & 0,00 & 30,00 & 60,00 & 55,26 & 24,20 & $-4,83$ & & & & \\
\hline \multirow{2}{*}{90} & 0,71 & 0,71 & 0,71 & 0,67 & 0,67 & 0,68 & \multirow{2}{*}{90,12} & \multirow{2}{*}{1,50} & \multirow{2}{*}{1,37} & \multirow{2}{*}{8,81} \\
\hline & 0,00 & 45,00 & 90,00 & 55,48 & 9,20 & $-34,64$ & & & & \\
\hline \multirow{2}{*}{120} & 0,71 & 0,50 & 0,71 & 0,68 & 0,47 & 0,68 & \multirow{2}{*}{120,11} & \multirow{2}{*}{1,25} & 115 & 834 \\
\hline & 0,00 & 60,00 & 120,00 & 55,68 & $-5,79$ & $-64,43$ & & & (1, & 0,34 \\
\hline 150 & 0,71 & 0,25 & 0,71 & 0,68 & 0,24 & 0,68 & 15008 & 106 & 098 & 746 \\
\hline 100 & 0,00 & 75,00 & 150,00 & 55,82 & $-20,78$ & $-94,26$ & 年 & 1,00 & & 8,40 \\
\hline 180 & 0,71 & 0,00 & 0,71 & 0,68 & 0,00 & 0,68 & 18003 & 100 & 092 & 68 \\
\hline & 0,00 & 90,00 & 180,00 & 55,85 & 44,00 & $-124,18$ & 100,03 & 1,00 & 0,92 & 1,04 \\
\hline
\end{tabular}

Table 1. Comparison of Ideal and Implemented Complex Amplitudes at output ports of the Beam Forming Network of 1 layer. 
The fig. 5 shows the calculate results for a beam-forming Network of 1 layer ( 2 in-ports and 3 out-ports) at 2.75 GHz. The phase shifter is at input port 2, instead at each output port, reducing the necessity of phase shifters in a ratio of 3:1. fig. $5 \mathrm{a}, \mathrm{b}$ and $\mathrm{c}$, shows the scanning angle of the radiation pattern calculated for the complex amplitudes at output ports of the beam forming network. The separation between radiant elements is set to $0.8 \lambda_{0}$ to reject the apparition of grating lobes in the results.

Table 1 compares ideal and implemented complex amplitudes for a structure of 1 layer at $2.75 \mathrm{GHz}$. The amplitudes and phases were calculated at the input of antennas. The results show that the phasing made by the implemented network is almost equal to the ideal beam-forming network for a given $\Delta \theta$. Also, table shows that the power delivered by the beamforming network is proportional to ideal one. The power difference is due to the power dissipated by chip resistors on isolated ports on the Gysel power dividers and the power losses by radiation of structure.

\section{CONCLUSION}

A new beam-forming network based on CORPS concepts is introduced. This new feed Network allows the reduction of complexity of beam-forming network, since it introduce a ratio reduction of Phase Shifters of N:1.

\section{REFERENCES}

[1] D. Betancourt, A. Ibañez and C. del Rio, “,Coherently Periodic Radiation Structures (CORPS): A Step Towards High Resolution Radiations Systems”, IEEE AP-S 2005, Washington, USA, July of 2005.

[2] D. Betancourt, A. Ibañez and C. del Rio, “,Designing Antenna Systems with CORPS (Coherently Periodic Radiation Structures)", $28^{\text {th }}$ ESA Antenna Workshop on Space Antenna Systems and Technologies, ESTEC, Noordwijk, Holland, June of 2005.

[3] R.C. Hansen, Microwave Scanning Antennas, Vol III: Array Systems, Academic Press, pag. 241-242, 1966.

[4] B. Ooi, "Compact EBG In Phase Hybrid-Ring Equal Power Divider," IEEE Trans. Microw. Theo. and Tech., Vol 53, No. 7, July 2005. 